\title{
A New Reconstruction Algorithm in Spline Signal Spaces
}

\author{
Chen Zhao, Yueting Zhuang, and Honghua Gan \\ College of Computer Science, Zhejiang University,Hangzhou 310027, P.R. China \\ csczhao@sohu.com, yzhuang@cs.zju.edu.cn
}

\begin{abstract}
In this research letter, we introduce a reconstruction formula in spline signal spaces which is a generalization of former results in [11. A general improved A-P iterative algorithm is presented. We use the algorithm to show reconstruction of signals from weighted samples and also show that the new algorithm shows better convergence than the old one. The explicit convergence rate of the algorithm is obtained.
\end{abstract}

\section{Introduction}

In the classical sampling problem, the reconstruction of $f$ on $\mathbb{R}^{d}$ from its samples $\left\{f\left(x_{j}\right): j \in J\right\}$, where $J$ is a countable indexing set, is one of main tasks in many applications in signal or image processing. However, this problem is ill-posed, and becomes meaningful only when the function $f$ is assumed to be bandlimited, or to belong to a shift-invariant space [1, 2, 3, 4, 8, 11, 12. For a bandlimited signal of finite energy, it is completely characterized by its samples, and described by the famous classical Shannon sampling theorem. Obviously, the shift-invariant space is not a space of bandlimited function unless the generator is bandlimited.

In many real applications, sampling points are not always regular. For example, the sampling steps need to be fluctuated according to the signals so as to reduce the number of samples and the computational complexity. If a weighted sampling is considered, the system will be made to be more efficient 1, 2, 3, 4, 5, 11, 12. It is well known that spline subspaces yield many advantages in their generation and numerical treatment so that there are many practical applications for signal or image processing. Therefore, the recent research of spline subspaces has received much attentions (see 3, 10, 11]).

For practical application and computation of reconstruction, Goh et al., showed practical reconstruction algorithm of bandlimited signals from irregular samples in [8], Aldroubi et al., presented a A-P iterative algorithm in [1,2, 4]. We will improve and generalize the A-P iterative algorithm and also show that the new algorithm shows better than the old one for convergence rate. That is, we can easy control the convergence rate of the algorithm with our requirement. At the same time, we don't increase the number of the sampling point. But this algorithm is not perfect. Because we immolate(increase) computation complexity as soon as improve convergence rate of the algorithm. 


\section{Reconstruction Algorithm in Spline Spaces}

By the special features of spline subspaces, we will present the new improved A-P algorithm and its convergence rate in spline spaces, which are more explicit. We introduce some notations and lemmas that will be used in this section.

The signal space $V_{N}=\left\{\sum_{k \in Z} c_{k} \varphi_{N}(\cdot-k):\left\{c_{k}\right\} \in \ell^{2}\right\}$ is spline space generated by $\varphi_{N}=\chi_{[0,1]} * \cdots * \chi_{[0,1]}(\mathrm{N}$ convolutions $), N \geq 1$.

Definition 2.1. A general bounded partition of unity(GBPU) is a set of function $\left\{\beta_{j_{1}}, \beta_{j_{2}}, \cdots, \beta_{j_{r}}\right\}$ that satisfy:

(1) $0 \leq \beta_{j_{1}}, \cdots, \beta_{j_{r}} \leq 1\left(\forall j_{1} \equiv j_{1}(j), \cdots, j_{r} \equiv j_{r}(j) \in J\right)$, where $J$ be countable separated index set.

(2) $\operatorname{supp} \beta_{j_{1}} \subset B_{\frac{\delta}{r}}\left(x_{j_{1}}\right), \cdots, \operatorname{supp} \beta_{j_{r}} \subset B_{\frac{\delta}{r}}\left(x_{j_{r}}\right)$,

(3) $\sum_{j \in J}\left(\beta_{j_{1}}+\cdots+\beta_{j_{r}}\right)=1$.

In fact, in the case of $r=1$, the above GBPU definition is ordinary BPU definition be used in [1,4].

We will assume that the weight function $\left\{\varphi_{x_{j}}: x_{j} \in X\right\}$ satisfy the following properties:

(i) $\operatorname{supp} \varphi_{x_{j}} \subset B_{\frac{a}{r}}\left(x_{j}\right)$

(ii) there exist $M^{r}>0$ such that $\int_{\mathbb{R}^{d}}\left|\varphi_{x_{j}}\right| d x \leq M$,

(iii) $\int_{\mathbb{R}^{d}} \varphi_{x_{j}} d x=1$

The operator $A$ and $Q$ defined by $A f=\sum_{j \in J}\left\langle f, \varphi_{x_{j_{1}}}\right\rangle \beta_{j_{1}}+\cdots+\left\langle f, \varphi_{x_{j_{r}}}\right\rangle \beta_{j_{r}}$ and $Q f(x)=\sum_{j} f\left(x_{j_{1}}\right) \beta_{j_{1}}(x)+\cdots+\sum_{j} f\left(x_{j_{r}}\right) \beta_{j_{r}}(x)$, respectively.

The other definitions and notations can be found in [1,4, 11, 12].

Lemma 2.1. [6] $\left\{\varphi_{N}(\cdot-k): k \in \mathbb{Z}\right\}$ is Riesz basis for $V_{N}, A_{N}=\sum_{k} \mid \hat{\varphi}_{N}(\pi+$ $2 k \pi)\left.\right|^{2}$ and $B_{N}=1$ are its lower and upper bounds, respectively.

Lemma 2.2. [4] If $\varphi$ is continuous and has compact support, then for any $f \in$ $V^{p}(\varphi)=\left\{\sum_{k \in \mathbb{Z}} c_{k} \varphi(\cdot-k):\left(c_{k}\right) \in \ell^{p}\right\}$, the following conclusions (i)-(ii) hold:

(i) $\|f\|_{L^{p}} \approx\|c\|_{\ell^{p}} \approx\|f\|_{W\left(L^{p}\right)}$,

(ii) $V^{p}(\varphi) \subset W_{0}\left(L^{p}\right) \subset W_{0}\left(L^{q}\right) \subset W\left(L^{q}\right) \subset L^{q}(\mathbb{R})(1 \leq p \leq q \leq \infty)$.

Lemma 2.3. If $f \in V_{N}$, then for any $0<\delta<1$ we have $\left\|\operatorname{osc}_{\delta}(f)\right\|_{L^{2}}^{2} \leq$ $(3 N \delta)^{2} \sum_{k \in \mathbb{Z}}\left|c_{k}\right|^{2}$, where osc $\delta(f)(x)=\sup _{|y| \leq \delta}|f(x+y)-f(x)|$.

Lemma 2.4. 4 For any $f \in V^{p}(\varphi)$, the following conclusions (i)-(ii) hold:

(i) $\left\|\operatorname{osc}_{\delta}(f)\right\|_{W\left(L^{p}\right)} \leq\|c\|_{\ell^{p}}\left\|\operatorname{osc}_{\delta}(\varphi)\right\|_{W\left(L^{1}\right)}$,

(ii) $\left\|\sum_{k \in \mathbb{Z}} c_{k} \varphi(\cdot-k)\right\|_{W\left(L^{p}\right)} \leq\|c\|_{\ell^{p}}\|\varphi\|_{W\left(L^{1}\right)}$. 
Lemma 2.5. If $X=\left\{x_{n}\right\}$ is increasing real sequence with $\sup _{i}\left(x_{i+1}-x_{i}\right)=\delta<1$, then for any $f=\sum_{k \in \mathbb{Z}} c_{k} \varphi_{N}(\cdot-k) \in V_{N}$ we have $\|Q f\|_{L^{2}} \leq\|Q f\|_{W\left(L^{2}\right)} \leq$ $\left(3+\frac{2 \delta}{r}\right)\|c\|_{\ell^{2}}\|\varphi\|_{W\left(L^{1}\right)}$.

Proof. For $f=\sum_{k \in \mathbb{Z}} c_{k} \varphi_{N}(\cdot-k)$ we have

$$
|f(x)-(Q f)(x)| \leq \operatorname{osc}_{\frac{\delta}{r}}(f)(x) .
$$

From this pointwise estimate and Lemma 2.2, 2.4, we get

$$
\begin{aligned}
\|f-Q f\|_{W\left(L^{2}\right)} & \leq\left\|o s c_{\frac{\delta}{r}}(f)\right\|_{W\left(L^{2}\right)} \\
& \leq\|c\|_{\ell^{2}}\left\|\operatorname{ssc}_{\frac{\delta}{r}}\left(\varphi_{N}\right)\right\|_{W\left(L^{1}\right)} .
\end{aligned}
$$

By the results of [1] or [4] we know

$$
\left\|\operatorname{osc}_{\frac{\delta}{r}}\left(\varphi_{N}\right)\right\|_{W\left(L^{1}\right)} \leq 2\left(1+\frac{\delta}{r}\right)\left\|\varphi_{N}\right\|_{W\left(L^{1}\right)} .
$$

Putting the above discussion together, we have

$$
\begin{aligned}
\|Q f\|_{L^{2}} & \leq\|Q f\|_{W\left(L^{2}\right)} \leq\|f-Q f\|_{W\left(L^{2}\right)}+\|f\|_{W\left(L^{2}\right)} \\
& \leq 2\left(1+\frac{\delta}{r}\right)\|c\|_{\ell^{2}}\left\|\varphi_{N}\right\|_{W\left(L^{1}\right)}+\left\|\sum_{k \in \mathbb{Z}} c_{k} \varphi_{N}(\cdot-k)\right\|_{W\left(L^{2}\right)} \\
& \leq 2\left(1+\frac{\delta}{r}\right)\|c\|_{\ell^{2}}\left\|\varphi_{N}\right\|_{W\left(L^{1}\right)}+\|c\|_{\ell^{2}}\left\|\varphi_{N}\right\|_{W\left(L^{1}\right)} \\
& \leq\left(3+\frac{2 \delta}{r}\right)\|c\|_{\ell^{2}}\left\|\varphi_{N}\right\|_{W\left(L^{1}\right)} .
\end{aligned}
$$

Theorem 2.1. Let $P$ be an orthogonal projection from $L^{2}(\mathbb{R})$ to $V_{N}$. If sampling set $X=\left\{x_{n}\right\}$ is a increasing real sequence with $\sup _{i}\left(x_{i+1}-x_{i}\right)=\delta<1$ and $\gamma=\frac{3 N \delta}{r \sqrt{\sum_{k}\left|\hat{\varphi}_{N}(\pi+2 k \pi)\right|^{2}}}<1$, then any $f \in V_{N}$ can be recovered from its samples $\left\{f\left(x_{j}\right): x_{j} \in X\right\}$ on sampling set $X$ by the iterative algorithm

$$
\left\{\begin{array}{l}
f_{1}=P Q f \\
f_{n+1}=P Q\left(f-f_{n}\right)+f_{n} .
\end{array}\right.
$$

The convergence is geometric, that is,

$$
\left\|f_{n+1}-f\right\|_{L^{2}} \leq \gamma^{n}\left\|f_{1}-f\right\|_{L^{2}} .
$$

Proof. By Lemma 2.1, Lemma 2.3 and properties of $\left\{\beta_{j 1}, \cdots, \beta_{j r}\right\}$, we have

$$
\begin{aligned}
\|(I-P Q) f\|_{L^{2}}^{2} & =\|P f-P Q f\|_{L^{2}}^{2} \leq\|P\|_{o p}^{2}\|f-Q f\|_{L^{2}}^{2}=\|f-Q f\|_{L^{2}}^{2} \\
& \leq\left\|o s c_{\frac{\delta}{r}}(f)\right\|_{L^{2}}^{2} \leq\left(3 N \frac{\delta}{r}\right)^{2} \sum_{k \in \mathbb{Z}}\left|c_{k}\right|^{2}=\left(3 N \frac{\delta}{r}\right)^{2}\|c\|_{\ell^{2}}^{2} \\
& \leq\left(\frac{3 N \delta}{r \sqrt{\sum_{k}\left|\hat{\varphi}_{N}(\pi+2 k \pi)\right|^{2}}}\right)^{2}\|f\|_{L^{2}}^{2} .
\end{aligned}
$$


Therefore

$$
\begin{aligned}
\left\|f_{n+1}-f\right\|_{L^{2}} & =\left\|f_{n}+P Q\left(f-f_{n}\right)-f\right\|_{L^{2}}=\left\|P Q\left(f-f_{n}\right)-\left(f-f_{n}\right)\right\|_{L^{2}} \\
& \leq\|I-P Q\|\left\|f-f_{n}\right\|_{L^{2}} \leq \cdots \leq\|I-P Q\|^{n}\left\|f-f_{1}\right\|_{L^{2}} .
\end{aligned}
$$

Combining with the estimate of $\|I-P Q\|$, we can imply

$$
\left\|f_{n+1}-f\right\|_{L^{2}} \leq \gamma^{n}\left\|f_{1}-f\right\|_{L^{2}}
$$

Taking assumption $\gamma=\frac{3 N \delta}{r \sqrt{\sum_{k}\left|\hat{\varphi}_{N}(\pi+2 k \pi)\right|^{2}}}<1$, we know the algorithm is convergent.

In the following, we will show the new improved A-P iterative algorithm from weighted samples in spline subspace.

Theorem 2.2. Let $P$ be an orthogonal projection from $L^{2}(\mathbb{R})$ to $V_{N}$ and weight function satisfy the following three conditions (i)-(iii):

(i) $\operatorname{supp} \varphi_{x_{j}} \subset\left[x_{j}-\frac{a}{r}, x_{j}+\frac{a}{r}\right]$

(ii) there exist $M>0$ such that $\int\left|\varphi_{x_{j}}(x)\right| d x \leq M$,

(iii) $\int \varphi_{x_{j}}(x) d x=1$.

If sampling set $X=\left\{x_{n}\right\}$ is a increasing real sequence with $\sup _{i}\left(x_{i+1}-x_{i}\right)=$ $\delta<1$ and we choose proper $\delta$ and a such that $\alpha=\frac{3 N}{r \sqrt{\sum_{k}\left|\hat{\varphi}_{N}(\pi+2 k \pi)\right|^{2}}}(\delta+$ $\left.a\left(3+\frac{2 a}{r}\right) M\right)<1$, then any $f \in V_{N}$ can be recovered from its weighted samples $\left\{\left\langle f, \varphi_{x_{j}}\right\rangle: x_{j} \in X\right\}$ on sampling set $X$ by the iterative algorithm

$$
\left\{\begin{array}{l}
f_{1}=P A f \\
f_{n+1}=P A\left(f-f_{n}\right)+f_{n} .
\end{array}\right.
$$

The convergence is geometric, that is,

$$
\left\|f_{n+1}-f\right\|_{L^{2}} \leq \alpha^{n}\left\|f_{1}-f\right\|_{L^{2}} .
$$

Proof. By $P f=f$ and $\|P\|_{o p}=1$, for any $f=\sum_{k \in \mathbb{Z}} c_{k} \varphi_{N}(\cdot-k) \in V_{N}$ we have

$$
\begin{aligned}
\|f-P A f\|_{L^{2}} & =\|f-P Q f+P Q f-P A f\|_{L^{2}} \\
& \leq\|f-Q f\|_{L^{2}}+\|Q f-A f\|_{L^{2}}
\end{aligned}
$$

From the proof of Theorem 2.1, we have the following estimate for $\|f-Q f\|_{L^{2}}$ :

$$
\|f-Q f\|_{L^{2}} \leq\left(\frac{3 N \delta}{r \sqrt{\sum_{k}\left|\hat{\varphi}_{N}(\pi+2 k \pi)\right|^{2}}}\right)\|f\|_{L^{2}} .
$$

For the second term $\|Q f-A f\|_{L^{2}}$ of (2) we have the pointwise estimate

$$
|(Q f-A f)(x)| \leq M Q\left(\sum_{k \in \mathbb{Z}}\left|c_{k}\right| \operatorname{osc}_{\frac{a}{r}}\left(\varphi_{N}\right)(x-k)\right) .
$$


From this pointwise estimate, Lemma 2.1, Lemma 2.3 and Lemma 2.5, it follows that:

$$
\begin{aligned}
\|Q f-A f\|_{L^{2}} & \leq M\left(3+\frac{2 a}{r}\right)\|c\|_{\ell^{2}}\left\|\operatorname{osc}_{\frac{a}{r}}\left(\varphi_{N}\right)\right\|_{W\left(L^{1}\right)} \\
& \leq M\left(3+\frac{2 a}{r}\right) \frac{\left\|o s c_{\frac{a}{r}}\left(\varphi_{N}\right)\right\|_{W\left(L^{1}\right)}}{\sqrt{\sum_{k}\left|\hat{\varphi}_{N}(\pi+2 k \pi)\right|^{2}}}\|f\|_{L^{2}} \\
& \leq M\left(3+\frac{2 a}{r}\right) \frac{3 N a}{r \sqrt{\sum_{k}\left|\hat{\varphi}_{N}(\pi+2 k \pi)\right|^{2}}}\|f\|_{L^{2}}
\end{aligned}
$$

By combining (3) and (6), we can obtain

$$
\|f-P A f\|_{L^{2}} \leq \frac{3 N}{r \sqrt{\sum_{k}\left|\hat{\varphi}_{N}(\pi+2 k \pi)\right|^{2}}}\left(\delta+a\left(3+\frac{2 a}{r}\right) M\right)\|f\|_{L^{2}},
$$

that is,

$$
\|I-P A\|_{L^{2}} \leq \frac{3 N}{r \sqrt{\sum_{k}\left|\hat{\varphi}_{N}(\pi+2 k \pi)\right|^{2}}}\left(\delta+a\left(3+\frac{2 a}{r}\right) M\right) .
$$

Similar to the procedure in the proof of Theorem 2.1, we have

$$
\left\|f_{n+1}-f\right\|_{L^{2}} \leq \alpha^{n}\left\|f_{1}-f\right\|_{L^{2}} .
$$

Remark 2.1. From the constructions of operator $Q$ and $A$, we know why item $r$ can appear in the convergence rate expression of the new improved algorithm. But $r$ is not appear in the old algorithm. Hence this algorithm improves the convergence rate of the old algorithm. In addition, it is obvious that we can easily control the convergence rate through choosing proper $r$ without changing sampling point gap $\delta$. That is, when $\delta$ and $a$ are proper given, we can obtain the convergence rate that we want through choosing proper $r$. We hope $r$ be enough large. But we increase the computation complexity as soon as choose larger $r$. So we should choose proper $r$ with our requirement.

\section{Conclusion}

In this research letter, we discuss in some detail the problem of the weighted sampling and reconstruction in spline signal spaces and provide a reconstruction formula in spline signal spaces, which is generalized and improved form of the results in [11]. Then we give general A-P iterative algorithm in general shiftinvariant spaces, and use the new algorithm to show reconstruction of signals from weighted samples. The algorithm shows better convergence than the old one. We study the new algorithm with emphasis on its implementation and obtain explicit convergence rate of the algorithm in spline subspaces. Due to the limitation of the page number, we omit some numerical examples, proofs of lemma and theorem and will show their detail in regular paper. 


\section{References}

1. A. Aldroubi. Non-uniform weighted average sampling and reconstruction in shiftinvariant and wavelet spaces. Appl. Comput. Harmon. Anal 13(2002)156-161.

2. A. Aldroubi, H. Feichtinger. Exact iterative reconstruction algorithm for multivate irregular sampled functions in spline-like spaces: The $L_{p}$ theory. Proc. Amer. Math. Soc 126(9)(1998)2677-2686.

3. A. Aldroubi, K. Gröchenig. Beurling-Landau-type theorems for non-uniform sampling in shift invariant spline spaces. J. Fourier. Anal. Appl, 6(1)(2000) 93-103.

4. A. Aldroubi and K. Gröchenig. Non-uniform sampling and reconstruction in shiftinvariant spaces. SIAM Rev 43(4)(2001)585-620.

5. W. Chen, S. Itoh and J. Shiki. On sampling in shift invariant spaces. IEEE Trans. Information. Theory 48(10)(2002)2802-2810.

6. C. K. Chui. An introduction to Wavelet, Academic Press, New York,1992.

7. H. G. Feichtinger. Generalized amalgams, with applications to Fourier transform. Can. J. of Math.,42(3)(1990)395-409

8. S. S. Goh, I. G. H. Ong. Reconstruction of bandlimited signals from irregular samples. Signal. Processing 46(3)(1995)315-329.

9. K. Gröchenig. Localization of frames, Banach frames, and the invertibility of the frame operator, J.Fourier.Anal.Appl 10(2)(2004)105-132.

10. W. C. Sun and X. W. Zhou. Average sampling in spline subspaces. Appl. Math. Letter, 15(2002)233-237.

11. J. Xian, S. P. Luo and W. Lin, Improved A-P iterative algorithm in spline subspaces. Lecture Notes in Comput. Sci, 3037(2004) 58-64.

12. J. Xian, X. F. Qiang. Non-uniform sampling and reconstruction in weighted multiply generated shift-invariant spaces. Far. East. J. Math. Sci, 8(3)(2003), 281-293. 